\title{
The Factors Associated with Good Responses to Speech Therapy Combined with Transcranial Direct Current Stimulation in Post-stroke Aphasic Patients
}

\author{
Il-Young Jung, M.D., Jong Youb Lim, M.D., Eun Kyoung Kang, M.D., Hae Min Sohn, S.T., \\ Nam-Jong Paik, M.D., Ph.D.
}

Department of Rehabilitation Medicine, Seoul National University College of Medicine, Seoul National University Bundang Hospital, Seongnam 463-707, Korea

Objective To determine factors associated with good responses to speech therapy combined with transcranial direct current stimulation (tDCS) in aphasic patients after stroke.

Method The language function was evaluated using Korean version of Western aphasia battery (K-WAB) before and after speech therapy with tDCS in 37 stroke patients. Patients received speech therapy for 30 minutes over 2 to 3 weeks (10 sessions) while the cathodal tDCS was performed to the Brodmann area 45 with $1 \mathrm{~mA}$ for 20 minutes. We compared the improvement of aphasia quotient \% (AQ\%) between two evaluation times according to age, sex, days after onset, stroke type, aphasia type, brain lesion confirmed by magnetic resonance image and initial severity of aphasia. The factors related with good responses were also checked.

Results AQ\% improved from pre- to post-therapy $(14.94 \pm 6.73 \%, \mathrm{p}<0.001)$. AQ\% improvement was greater in patients with less severe, fluent type of aphasia who received treatment before 30 days since stroke was developed $(\mathrm{p}<0.05)$. The adjusted logistic regression model revealed that patients with hemorrhagic stroke were more likely to achieve good responses (odds ratio $=4.897, \mathrm{p}<0.05$ ) relative to infarction. Initial severity over $10 \%$ in AQ\% was also found to be significantly associated with good improvement (odds ratio=8.618, $\mathrm{p}<0.05$ ).

Conclusion Speech therapy with tDCS was established as a treatment tool for aphasic patients after stroke. Lower initial severity was associated with good responses.

Key Words Stroke, Aphasia, Speech therapy, Recovery, Transcranial direct current stimulation

Received May 3, 2010; Accepted March 22, 2011

Corresponding author: Nam-Jong Paik

Department of Rehabilitation Medicine, Seoul National University College of Medicine, Seoul National University Bundang Hospital, 166, Gumi-ro, Bundang-gu, Seongnam 463-707, Korea

Tel: +82-31-787-7731, Fax: +82-31-712-3913, E-mail: njpaik@snu.ac.kr

(c) This is an open-access article distributed under the terms of the Creative Commons Attribution Non-Commercial License (http://creativecommons.org/ licenses/by-nc/3.0) which permits unrestricted noncommercial use, distribution, and reproduction in any medium, provided the original work is properly cited. Copyright $\odot 2011$ by Korean Academy of Rehabilitation Medicine

\section{INTRODUCTION}

Stroke is the leading cause of chronic disability in adults, and is a leading public health problem in Korea in terms of the socioeconomic burden of more than 3 trillion won per year. ${ }^{1}$ More than $60 \%$ of stroke patients have persistent neurological deficits, with various disabilities 
of daily living, aphasia, neglect, and other cognitive impairment. ${ }^{2,3}$ Among other factors, aphasia interrupts communication with therapists, making it difficult to participate in rehabilitation sessions. According to the brain lesion, aphasia is expressed as several types, and the recovery rates are diverse..$^{4-7}$ As an effort to improve cognitive impairment including aphasia, different treatment strategies have emerged. Neurorehabilitative treatment is one of those strategies and undergoing research and development.

Generally, the treatment of aphasia is based on speech therapy for the improvement of communication. A brain polarization of the form of transcranial direct current stimulation (tDCS), which was the method for enhancing language function and attention, has been proposed as an adjuvant strategy to improve aphasia in neurorehabilitation. ${ }^{8,9}$ As a tool for non-invasive brain polarization tDCS, does not cause pain and can easily be applied to sham stimulation, because it cannot be detected through auditory and somatosensory senses. In addition, it is known that brain excitability is controlled with tDCS depending on the polarity. ${ }^{10}$ Lazar et al. ${ }^{11}$ reported that spontaneous recovery mechanisms during the first 90 days may be augmented with biologically focused intervention early after stroke, perhaps with noninvasive brain stimulation, pharmacology, or targeted behavioral methods to improve function beyond what is currently predicted.

With the study on adjuvant therapy such as tDCS, studies of factors affecting recovery of aphasia are also progressing. ${ }^{12}$ Previous studies reported that prognosis is not better for non-fluent aphasia compared to fluent aphasia, ${ }^{13}$ and predictability is less but the degree of improvement changed by the severity of stroke or aphasia. ${ }^{14-16}$ According to studies in 2010, language dysfunctions after stroke seem to show highly predictable recovery which is related to initial impairments and $70 \%$ of patients recovered during the first 90 days. ${ }^{11,17}$

To improve the effectiveness of speech therapy in poststroke patients with aphasia, this study compared the functional change of language after speech therapy combined with tDCS and the factors associated with functional improvement. In particular, it evaluated the factors associated with good responses to speech therapy combined with tDCS. Additionally, we confirmed that speech therapy combined with tDCS was a successful method to improve therapeutic effectiveness in aphasia after stroke and was not inferior to conventional speech therapy.

\section{MATERIALS AND METHODS}

\section{Subjects}

Our study used medical records of image-verified patients, who were diagnosed and received treatments for aphasia after stroke between December 2007 and April 2009, and underwent assessment with Korean version of Western aphasia battery (K-WAB) before and after speech therapy combined with tDCS through in- or outpatients clinic. Subjects were excluded if they did not receive speech therapy combined with tDCS, did not complete speech therapy 10 times, or were treated less than 2 times a week. Patients were also excluded from the study if they had headache after tDCS, had a skull defect due to surgery, such as craniectomy, and those with contraindications to tDCS. A retrospective medical records survey was performed with a total 118 patients, and 67 patients were excluded. Four were discontinued because of discomfort such as headache, and 10 transferred to other hospitals or were discharged during treatment (Fig. 1). A total of 37 subjects with aphasia (males 26, females 11 , mean age $62.4 \pm 12.9$ year-old) were included at the analysis stage. The subjects started speech therapy combined with tDCS at an average of 220.9 days after stroke (Table 1 ).

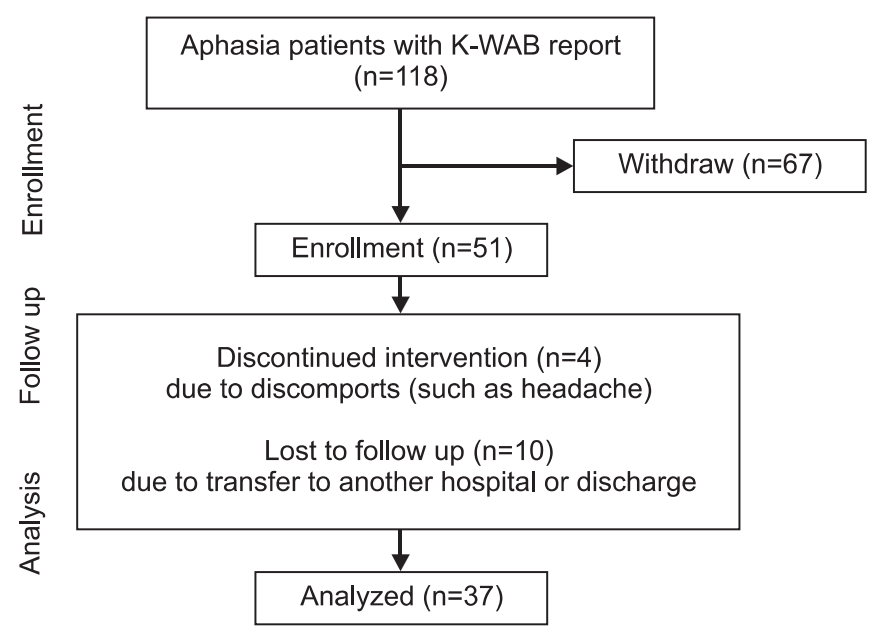

Fig. 1. Flowchart. K-WAB: Korean version of Western aphasia battery. 
Table 1. Characteristics of Patients and Initial AQ\% According to Demographic Variables and Stroke Features

\begin{tabular}{|c|c|c|c|c|}
\hline Variables & & No. $(n=37)$ & Initial AQ\% & p-value \\
\hline \multirow[t]{2}{*}{ Age (years) } & $>65$ & 22 & $20.79 \pm 21.26$ & 0.949 \\
\hline & $\leq 65$ & 15 & $26.00 \pm 20.52$ & \\
\hline \multirow[t]{2}{*}{ Sex } & Male & 26 & $20.94 \pm 19.09$ & 0.328 \\
\hline & Female & 11 & $27.55 \pm 24.85$ & \\
\hline \multirow[t]{4}{*}{ Days from onset } & $\leq 30$ & 13 & $22.92 \pm 21.75$ & 0.977 \\
\hline & $>30$ & 24 & $22.89 \pm 20.80$ & \\
\hline & $\leq 90$ & 27 & $23.01 \pm 18.37$ & 0.234 \\
\hline & $>90$ & 10 & $22.60 \pm 27.59$ & \\
\hline \multirow[t]{2}{*}{ Stroke type } & Infarction & 20 & $17.57 \pm 15.84$ & 0.813 \\
\hline & Hemorrhage & 16 & $25.31 \pm 19.26$ & \\
\hline \multirow[t]{2}{*}{ Aphasia type } & Fluent & 10 & $43.60 \pm 26.29$ & $0.001^{*}$ \\
\hline & Non-fluent & 26 & $14.55 \pm 11.33$ & \\
\hline \multirow[t]{8}{*}{ Brain MRI (overlab) } & Broca's area $(+)$ & 13 & $12.08 \pm 7.14$ & $0.022^{*}$ \\
\hline & Broca's area (-) & 24 & $28.77 \pm 23.47$ & \\
\hline & Wernicke's area (+) & 15 & $12.56 \pm 13.77$ & 0.218 \\
\hline & Wernicke's area (-) & 22 & $29.95 \pm 22.12$ & \\
\hline & Arcuate fasciculus (+) & 21 & $16.24 \pm 17.57$ & 0.382 \\
\hline & Arcuate fasciculus (-) & 16 & $31.65 \pm 22.06$ & \\
\hline & Insular area (+) & 28 & $17.94 \pm 13.26$ & $0.002^{*}$ \\
\hline & Insular area (-) & 9 & $38.33 \pm 31.73$ & \\
\hline
\end{tabular}

Values are mean \pm standard deviation

AQ: Aphasia quotient

${ }^{*} \mathrm{p}<0.05$ by independent $\mathrm{t}$-test

\section{Methods}

Speech therapy was performed based on the type of aphasia, specific features, and various responses of stimulation. One skillful speech therapist analyzed the results of K-WAB and individual aphasia types. The speech therapy methods used were stimulation with auditory and visual sense, such as a melody intonation therapy, visual action therapy, and auditory comprehension training. There were also other methods of speech therapy, such as context and stimulation word-oriented therapy, therapy for promoting aphasics' communicative effectiveness to improve communication skills, and practice sessions of speaking using a cognitive therapeutic approach. A cathodal tDCS (Iomed Phoresor PM850, Vista, Salt Lake City, Utah, USA) combined with speech therapy was applied to the patients. Thirty five $\mathrm{cm}^{2}$ saline-soaked anodal and cathodal electrodes (about $6 \times 6 \mathrm{~cm}$ ) were placed on the Broadmann area 45 (According to 10-20 electroencephalography system, Broadmann area 45 was defined as the space between T4-Fz and F8-Cz.) of una- ffected cerebral cortex overlying the inferior frontal gyrus and on the contralateral forehead above the orbit, as described previously. ${ }^{10}$ The direct current was initially increased in a ramp-like fashion over several seconds (010 seconds) until reaching $1 \mathrm{~mA}$ (current density of 0.03 $\mathrm{mA} / \mathrm{cm}^{2}$ ). In tDCS, stimulation was maintained for a total of 20 minutes. ${ }^{10,18}$ Standard speech therapy combined with tDCS was for 30 minutes, a total of 10 times. In principle, speech therapy combined with tDCS was performed 5 times a week for two weeks, and at least 3 times a week for three weeks to consider the situation of the patient. For comparison of therapeutic effects, K-WAB was checked before speech therapy combined with tDCS and after the end of 10 sessions.

The aphasia quotient \% (AQ\%) was used as a primary outcome measure, and factors such as age, sex, initial treatment time after stroke, types of stroke, and type of aphasia were associated with the improvement of AQ\% and investigated by reviewing medical records. However, a functional state index of patients was not used because 
of significant missing data. Invasion of brain lesionrelated speech function was assessed by the axial image generated from magnetic resonance imaging (MRI) of the brain from each patient and categorized as Broca's area, Wernicke's area, arcuate fasciculus, and insula. ${ }^{19-21}$

Depending on the previously reported aphasia type of Koreans, ${ }^{22}$ the standard value of primary initial severity assessment for analysis was determined to be $10 \%$ of $\mathrm{AQ} \%(\mathrm{AQ} \%<10 \%$ or $\mathrm{AQ} \% \geq 10 \%)$ and the standard value of secondary initial severity assessment was determined to be $20 \%$ (AQ\% $<20 \%$ or $A Q \% \geq 20 \%$ ). A good response group was also defined as the group with $7 \%$ more than the degree of improvement in AQ\% compared with K-WAB results before and after speech therapy.

\section{Statistics}

Statistical Package for the Social Sciences (SPSS, version 17.0 for Windows, Chicago, USA) was used for all statistical analysis. Significance was accepted at $\mathrm{p}<0.05$ and $95 \%$ confidence intervals (CI) were provided. Evaluated AQ\% of K-WAB before speech therapy combined with tDCS was compared between groups and variable factors, such as age, sex, days from onset, type of stroke, aphasia type, brain lesion, initial severity, by means of independent t-test, paired t-test, and logistic regression. Changes across therapy in each factor of K-WAB were evaluated by a paired t-test, and an independent t-test was used for delta $\mathrm{AQ} \%(\triangle \mathrm{AQ} \%)$ before and after K-WAB evaluation separated by previous factors. The independent effects of speech therapy combined with tDCS on functional recovery were assessed by a multivariate logistic regression model, unadjusted or adjusted for 7 key prognostic factors, namely, age, sex, days from onset, stroke type (infarction or hemorrhage), aphasia type, lesion of brain
MRI (Broca's area, Wernicke's area, arcuate fasciculus, and insula), and initial severity. The correlation between the AQ\% improvements of taking the natural logarithm with initial severity was evaluated by $\mathrm{R}^{2}$ and Pearson correlation coefficient.

\section{RESULTS}

\section{Initial AO\% before speech therapy with tDCS}

There was no statistically significant different in the $\mathrm{AQ} \%$ of patients between sex $(\mathrm{p}=0.328)$ and age $(\mathrm{p}=0.949)$ for over 65 year-olds compared with under 65 year-olds before speech therapy combined tDCS. No significant differences were found between AQ\% treated at $\leq 30$ days with $>30$ days $(\mathrm{p}=0.977$ ), and at $\leq 90$ days and $>90$ days $(p=0.234)$ after stroke. No statistical difference was observed between types of stroke $(p=0.813)$. The type of aphasia could be categorized as non-fluent aphasia, such as global aphasia, transcortical motor aphasia, motor aphasia or Broca's aphasia, and fluent aphasia, such as transcortical sensory aphasia, sensory aphasia, Wernicke's aphasia, anomic aphasia, or conduction aphasia. When AQ\% was compared for these two types of aphasia, the initial AQ\% of fluent aphasia was significantly higher than one of the non-fluent aphasia, which was reported to be the same as the existing data $(\mathrm{p}=0.001)$. Brain lesions were individually separated by Broca's area corresponding to Broadmann area 45, Wernicke's area corresponding to posterior Broadmann area 22, arcuate fasciculus connecting the Broca's and Wernicke's area, and insula located on the inside of the temporal lobe using MRI FLAIR or T2 weighted axial image. When involving Broca's area, initial AQ\% was significantly lower than non-involving Broca's area following previous

Table 2. Improvement of K-WAB Items from Pre- to Post-Evaluation

\begin{tabular}{lcccc}
\hline & Pre-evaluation & Post-evaluation & Correlation & p-value \\
\hline Fluency $(\mathrm{n}=37)$ & $19.65 \pm 21.77$ & $33.22 \pm 28.82$ & 0.616 & $<0.001$ \\
Auditory comprehension $(\mathrm{n}=37)$ & $36.49 \pm 26.59$ & $49.22 \pm 29.11$ & 0.819 & $<0.001$ \\
Repetition $(\mathrm{n}=37)$ & $23.73 \pm 23.61$ & $38.00 \pm 27.45$ & 0.863 & $<0.001$ \\
Naming $(\mathrm{n}=37)$ & $24.84 \pm 22.60$ & $39.65 \pm 28.11$ & 0.823 & $<0.001$ \\
Reading $(\mathrm{n}=34)$ & $30.44 \pm 29.15$ & $46.21 \pm 32.29$ & 0.848 & $<0.001$ \\
Writing $(\mathrm{n}=24)$ & $38.21 \pm 22.38$ & $53.29 \pm 26.40$ & 0.813 & $<0.001$ \\
Total & $22.92 \pm 20.84$ & $36.86 \pm 27.57$ & 0.795 & $<0.001$ \\
\hline
\end{tabular}

Values are mean \pm standard deviation

K-WAB: Korean version of Western aphasia battery 
classification $(\mathrm{p}=0.022)$. When involving insula area, initial AQ\% was also statistically significant lower than non-involving insula area $(\mathrm{p}=0.002$, Table 1$)$.

Improvement of K-WAB results after speech therapy with tDCS

AQ\% was improved after speech therapy combined with tDCS (before $22.92 \pm 20.84 \%$, after $36.86 \pm 27.57 \%$, p $<0.001$ ). Each item, such as fluency, auditory comprehension, repetition, naming, reading or writing significantly improved $(\mathrm{p}<0.001$, Table 2$)$.

\section{Change of $\mathrm{AQ} \%$ according to factors}

When the initiation time of therapy was divided into two groups for 30 days after stroke, change of AQ\% ( $\triangle \mathrm{AQ} \%$ ) significantly increased for less than 30 days $(\mathrm{p}=0.001)$ compared to over 30 days. In aphasia type, $\triangle \mathrm{AQ} \%$ of the fluent aphasia increased more than one of the nonfluent aphasia ( $\mathrm{p}=0.022)$. In the cases of initial severity, $\triangle \mathrm{AQ} \%$ significantly increased in both of the initial AQ\% above $10 \%$ or $20 \%(10 \% \mathrm{p}=0.030,20 \% \mathrm{p}<0.001)$ rather than under $10 \%$ or 20 . According to age and sex, however, $\triangle \mathrm{AQ} \%$ showed no significant change. No statistical differences were found between the starting time based on 90 days after stroke, stroke type, and classification for brain lesion using MRI (Table 3).

Factors associated with good responses

Odds ratios of factors related with induced aphasia were statistically confirmed. When infarction was the standard,

Table 3. Improvement of AQ\% According to Demographic Variables and Stroke Features

\begin{tabular}{|c|c|c|c|c|}
\hline Variables & & No. & $\Delta \mathrm{AQ \%}$ & p-value \\
\hline \multirow[t]{2}{*}{ Age (years) } & $>65$ & 22 & $13.00 \pm 16.20$ & 0.612 \\
\hline & $\leq 65$ & 15 & $15.33 \pm 18.03$ & \\
\hline \multirow[t]{2}{*}{ Sex } & Male & 26 & $12.12 \pm 14.41$ & 0.053 \\
\hline & Female & 11 & $18.27 \pm 21.50$ & \\
\hline \multirow[t]{4}{*}{ Days from onset } & $\leq 30$ & 13 & $21.69 \pm 21.19$ & $0.001^{*}$ \\
\hline & $>30$ & 24 & $9.75 \pm 12.37$ & \\
\hline & $\leq 90$ & 27 & $16.33 \pm 17.30$ & 0.161 \\
\hline & $>90$ & 10 & $7.50 \pm 13.99$ & \\
\hline \multirow[t]{2}{*}{ Stroke type } & Infarction & 20 & $10.15 \pm 15.40$ & 0.162 \\
\hline & Hemorrhage & 16 & $19.44 \pm 17.69$ & \\
\hline \multirow[t]{2}{*}{ Aphasia type } & Fluent & 10 & $21.00 \pm 21.40$ & $0.022^{*}$ \\
\hline & Non-fluent & 26 & $11.77 \pm 14.39$ & \\
\hline \multirow[t]{8}{*}{ Brain MRI (overlab) } & Broca's area (+) & 13 & $13.54 \pm 16.87$ & 0.575 \\
\hline & Broca's area (-) & 24 & $14.17 \pm 17.06$ & \\
\hline & Wernicke's area (+) & 15 & $9.07 \pm 14.39$ & 0.272 \\
\hline & Wernicke's area (-) & 22 & $17.27 \pm 17.74$ & \\
\hline & Arcuate fasciculus (+) & 21 & $13.43 \pm 15.74$ & 0.680 \\
\hline & Arcuate fasciculus (-) & 16 & $14.63 \pm 18.51$ & \\
\hline & Insular area $(+)$ & 28 & $15.32 \pm 17.54$ & 0.344 \\
\hline & Insular area (-) & 9 & $9.67 \pm 14.08$ & \\
\hline \multirow[t]{4}{*}{ Initial severity (\%) } & $\mathrm{AQ} \%<10$ & 12 & $7.92 \pm 11.63$ & $0.030^{*}$ \\
\hline & $A Q \% \geq 10$ & 25 & $16.84 \pm 18.23$ & \\
\hline & $\mathrm{AQ} \%<20$ & 18 & $8.22 \pm 9.70$ & $<0.001^{*}$ \\
\hline & $\mathrm{AQ} \% \geq 20$ & 19 & $19.37 \pm 20.22$ & \\
\hline
\end{tabular}

Values are mean \pm standard deviation

$\triangle \mathrm{AQ} \%$ = post-evaluation $\mathrm{AQ} \%$ - pre-evaluation $\mathrm{AQ} \%$

AQ: Aphasia quotient

${ }^{*} \mathrm{p}<0.05$ by independent $\mathrm{t}$-test 
unadjusted odds ratio of stroke type was 5.571. This meant a degree of improvement in language function, implying hemorrhage was 5.5 times higher than infarction $(\mathrm{p}=$ 0.021 ) after speech therapy with tDCS. In adjusting for initial severity of odds ratio, the degree of improvement was also 4.9 times higher $(p=0.037)$. Initial $A Q \% \geq 10 \%$ was the factor associated with good responses after speech therapy, in which the degree of improvement was 5.3 times higher compared with initial AQ\%<10\% ( $p=0.033)$. In adjusting for initial severity of odds ratio, the degree of improvement was also 8.6 times higher $(\mathrm{p}=0.029$, Table 4). In addition, initial AQ\% was significantly correlated with the natural logarithm of improvement of AQ\% after therapy $\left(\mathrm{R}^{2}=0.286\right.$, Pearson correlation coefficient $=0.536$, $\mathrm{p}=0.002$, Fig. 2).

\section{DISCUSSION}

In this study, the initial severity was significantly different between more than $10 \%(7.92 \%)$ and less than $10 \%(16.84 \%)$, and the difference was significant even if divided by $20 \%$ using t-test. We confirmed that the higher the initial severity, the lower the $\Delta \mathrm{AQ} \%$ by logistic regression. Initial severity as a single factor supported the most important prognostic factor. To achieve these results, however, criteria for good responses after therapy

Table 4. Unadjusted and Adjusted Odds Ratios (OR) of Good Responses ( $\triangle \mathrm{AQ} \% \geq 7 \%$ )

\begin{tabular}{|c|c|c|c|c|c|c|}
\hline Variables & & No. & 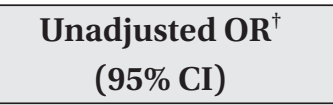 & p-value & $\begin{array}{c}\text { Adjusted } \mathrm{OR}^{\dagger} \\
(95 \% \mathrm{CI})\end{array}$ & p-value \\
\hline \multirow[t]{2}{*}{ Age (years) } & $>65$ & 22 & 1.000 & & 1.000 & \\
\hline & $\leq 65$ & 15 & $1.800(0.476-6.812)$ & 0.387 & $1.713(0.446-6.575)$ & 0.433 \\
\hline \multirow[t]{2}{*}{ Sex } & Male & 26 & 1.000 & & 1.000 & \\
\hline & Female & 11 & $1.200(0.292-4.935)$ & 0.800 & $1.115(0.264-4.702)$ & 0.883 \\
\hline \multirow[t]{4}{*}{ Days from onset } & $\leq 30$ & 13 & $1.891(0.478-7.486)$ & 0.364 & $1.909(0.477-7.638)$ & 0.361 \\
\hline & $>30$ & 24 & 1.000 & & 1.000 & \\
\hline & $\leq 90$ & 27 & $3.394(0.717-16.073)$ & 0.124 & $3.461(0.715-16.745)$ & 0.123 \\
\hline & $>90$ & 10 & 1.000 & & 1.000 & \\
\hline \multirow[t]{2}{*}{ Stroke type } & Infarction & 20 & 1.000 & & 1.000 & \\
\hline & Hemorrhage & 16 & $5.571(1.297-23.934)$ & $0.021^{*}$ & $4.897(1.104-21.713)$ & $0.037^{*}$ \\
\hline \multirow[t]{2}{*}{ Aphasia type } & Non-fluent & 26 & 1.000 & & 1.000 & \\
\hline & Fluent & 10 & $2.722(0.574-12.914)$ & 0.207 & $2.690(0.368-19.631)$ & 0.329 \\
\hline \multirow[t]{8}{*}{ Brain MRI (overlab) } & Broca's area $(+)$ & 13 & 1.000 & & 1.000 & \\
\hline & Broca's area (-) & 24 & $1.379(0.356-5.341)$ & 0.642 & $1.159(0.267-5.033)$ & 0.844 \\
\hline & Wernicke's area (+) & 15 & 1.000 & & 1.000 & \\
\hline & Wernicke's area (-) & 22 & $3.500(0.072-1.137)$ & 0.075 & $3.528(0.774-16.073)$ & 0.103 \\
\hline & Arcuate fasciculus (+) & 21 & 1.000 & & 1.000 & \\
\hline & Arcuate fasciculus (-) & 16 & $1.414(0.383-5.227)$ & 0.603 & $1.212(0.295-4.973)$ & 0.789 \\
\hline & Insular area $(+)$ & 28 & 1.000 & & 1.000 & \\
\hline & Insular area (-) & 9 & $1.250(0.276-5.653)$ & 0.772 & $0.984(0.185-5.228)$ & 0.984 \\
\hline \multirow[t]{4}{*}{ Initial severity (\%) } & $\mathrm{AQ} \%<10$ & 12 & 1.000 & & 1.000 & \\
\hline & $\mathrm{AQ} \% \geq 10$ & 25 & $5.333(1.142-24.899)$ & $0.033^{*}$ & 8.618 (1.248-59.528) & $0.029^{*}$ \\
\hline & $\mathrm{AQ} \%<20$ & 18 & 1.000 & & 1.000 & \\
\hline & $\mathrm{AQ} \% \geq 20$ & 19 & $2.694(0.713-10.178)$ & 0.144 & $3.753(0.553-25.494)$ & 0.176 \\
\hline
\end{tabular}

$\Delta \mathrm{AQ} \%=$ post-evaluation $\mathrm{AQ} \%$ - pre-evaluation $\mathrm{AQ} \%$

CI: Confidence interval, AQ: Aphasia quotient

${ }^{*} \mathrm{p}<0.05$ by logistic regression analysis

${ }^{\dagger}$ Unadjusted odds ratios by logistic regression analysis. ${ }^{\ddagger}$ Adjusted odds ratios by multivariate logistic regression analysis; adjusted for all other variables in model 


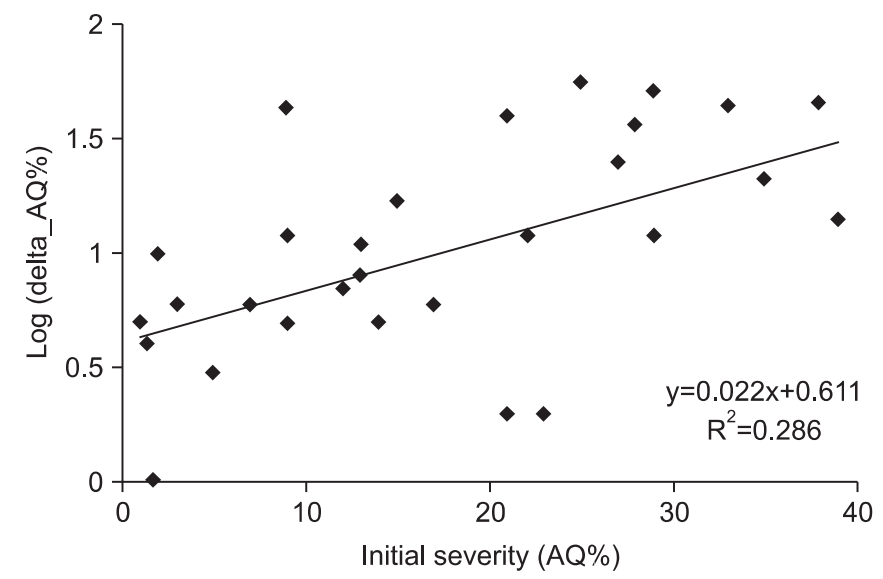

Fig. 2. Initial severity correlated with the natural logarithm of improvement of $\mathrm{AQ} \%$. $\mathrm{R}^{2}=0.286$, Pearson correlation coefficient $=0.536(\mathrm{p}=0.002)$. Delta $\mathrm{AQ} \%=$ post-evaluation AQ\%-pre-evaluation AQ\%, AQ\%: Aphasia quotient \%.

and the definition of severity of aphasia were necessary. In previous studies, the criteria for dividing the severity of aphasia were not clearly defined. Depending on previously reported aphasia type for Koreans, initial severity was decided by AQ\%. Compared to different kinds of aphasia, global aphasia had significantly lower AQ\%, which meant a higher severity of aphasia. Kang et al. ${ }^{22}$ reported that $\mathrm{AQ} \%$ of global aphasia was $7.6 \pm 8.2 \%$, $\mathrm{AQ} \%$ of mixed transcortical aphasia was $19.5 \pm 12.0 \%$, AQ\% of Broca's aphasia was $26.6 \pm 16.4 \%$, and AQ\% of Wernicke's aphasia was $32.1 \pm 11.9 \%$ in the Korean population. Standard value of primary severity assessment was determined as $10 \%$ by AQ\% of global aphasia. Secondary standard value was also determined as $20 \%$ by AQ\% of mixed transcortical aphasia, which was the partially recovered form of global aphasia. In addition, there were no reports for the definition of a good response, and no data that could set a standard. In this study, the good response group was defined as $7 \%$ degree of improvement by AQ\% difference $(7.1 \%)$ between mixed transcortical aphasia, the boundary between high and low severity aphasia among high severity, and Broca's aphasia, the boundary between high and low severity aphasia among the low severity.

No significant difference was found between initial severities evaluated with K-WAB in patients treated within 30 days and patients treated 30-90 days after stroke onset. $^{22}$ We also used 30 and 90 days to classify the patients and adjusted for initial severities, and found no difference in the risks between two groups using the odds ratio. One of the problems in studying aphasia is that it can be hard to distinguish between spontaneous recoveries that often take place in the initial months and the response to speech therapy. ${ }^{23}$ This may be explained by studies on aphasia recovery, which mostly focus on acute or subacute stroke patients, whereas studies on response to treatment are mostly based on patients at chronic stages. ${ }^{24-26}$ In this study, patients treated within 30 days of stroke onset showed significantly higher improvements in the $A Q \%$ compared to those treated after 30 days, which may be the effect of initial spontaneous recovery. When compared with the results from Lazar et al. ${ }^{11}$, however, even higher improvements in the AQ score were seen in our results, despite the time difference of initial evaluation. Lazar et al. ${ }^{11}$ performed Western aphasia battery (WAB) to acute stroke patients initially without speech therapy and 90 days afterwards, and found that the the AQ score improved from 20.0 \pm 7.7 to $27.5 \pm 3.7(\mathrm{p}<0.001)$. In this study, 33 patients were evaluated using K-WAB after an average period of 70 days of onset, and showed an improvement of AQ score by $15.8 \pm 16.9$, from $29.7 \pm 21.6$ initially to $45.5 \pm 28.2$ after 10 sessions of combined tDCS and speech therapy for 2 to 3 weeks. This is a greater improvement compared to other reports, in which the improvement of AQ score without speech therapy $(\mathrm{p}<0.02)$ can lead to the assumption that tDCS combined with speech therapy in addition to spontaneous recovery contributed to the improvement in speech function. Patients in this study were at chronic stages, so the greater improvement in the AQ score showed the effect of tDCS combined with speech therapy. Furthermore, another study on aphasia treatment, where the patients received advanced community-based treatment programs (average time taken to treatment from onset was $68.6 \pm 12.3$ days), showed an overall improvement of AQ score from $42.5 \pm 27.4$ to $51.6 \pm 28.7$. Although not statistically proven, this is presumed to be a lesser improvement than our study. ${ }^{27}$

In this study, aphasia due to hemorrhage rather than infarction was a statistically significant related factor for improvement in language functions. This has not been reported in previous studies, because instead of classifying the patients by lesions in the cortex or subcortex, we classified them into patients with hemorrhage or infarct. Patients with hemorrhage mostly had 
lesions in the basal ganglia, capsule and corona radiata, which were classified as the subcortical area. Compared to infarction, hemorrhage had less effect on the cortical areas, and thereby was shown to be a better prognostic factor. ${ }^{20,22}$ However, this was in line with the results of this study since patients with cortical lesions were likely to have higher severity of aphasia than patients with subcortical lesions. When the brain lesions were classified into language areas, the odds ratio was not statistically significant. This can be explained by the fact that patients who had aphasia due to hemorrhage began therapy in 149.5 days, which is earlier than patients with aphasia due to infarction (244.3 days). Although not shown due to missing data, better overall function, excluding aphasia, in hemorrhage patients than infarct patients may have contributed to the above result.

Among fluent and non-fluent aphasia divided by brain lesion, fluent aphasia was known to get a good prognosis. However, a previous study had reported that the degree of improvement, which was different the prognosis in global aphasia is greater than Wernicke's aphasia. ${ }^{16}$ Based on this report, looking at the results of this study, $\mathrm{AQ} \%(43.60 \pm 26.29 \%)$ of fluent aphasia was $30 \%$ higher than AQ\% (14.55 $\pm 11.33 \%)$ of non-fluent aphasia. In fluent aphasia, the maximum value of AQ\% increased as symptom improvement was higher. However, the absolute AQ\% value of improvement in non-fluent aphasia was higher, because very low initial AQ\% in nonfluent aphasia may be significantly changed. Therefore, it is considered that there was no statistically significant change in the improvement of AQ\% between fluent and non-fluent aphasia.

Transcranial direct current stimulation (tDCS) is reported to have the ability to control resting membrane potentials in neurons of the brain and generate depolarization or hyperpolarization depending on the position and polarity of the electrode.$^{28}$ How tDCS affects the function of language, however, is still controversial, and precise stimulation of the targeted area of brain needs to be investigated. Whether tDCS combined with speech therapy has a treatment response in aphasia has not been reported. However, the safety of tDCS was validated in a study using a double-blind, sham-controlled method, ${ }^{10}$ and another study showed improved accuracy in naming task with anodal stimulation of the Broca's area. ${ }^{9}$ Anodal tDCS over the left prefrontal cortex has been reported to enhance naming accuracy in chronic aphasic patients, ${ }^{29}$ while stimulation of the Wernicke's area, and other cathodal stimulation also showed improvements in naming accuracy in other reports. ${ }^{29,30}$

This study showed significant improvements in all items of K-WAB, which was inconsistent with previously mentioned studies reporting improvements in naming accuracy. This may be the result of speech therapy rather than the effect of cathodal tDCS. Cathodal tDCS can generate hyperpolarization by changing the resting potential of cell membranes, and inhibit activation of cells thereby affecting the recovery of function of the contralateral side. Therefore, tDCS may contribute to creating a sound environment for speech therapy, but cannot be the fundamental speech therapy itself. Most studies used naming performances such as the Boston naming test, picture naming task, and overt naming task as their dependent variable when evaluating normal or aphasic patients after tDCS, which was a possible explanation for the limited results of improvements in the naming accuracy only. Using the WAB or K-WAB as dependent variables will allow more comprehensive evaluation of the speech performance.

This study had several limitations. The patients ranged from acute to chronic stages, and initial evaluation time varied among patients. The study was based on retrospective review of medical records, so the patients could not be adjusted for factors that could possibly affect aphasia if they had no results for other cognitive dysfunction. Structural components that might affect the prognosis, such as severe brain atrophy, were not taken into account when reviewing brain MRIs of the patients, and this could explain why patients achieved less AQ\% improvements than expected. An objective measure to identify the effect of tDCS on improvement of speech function was not taken due to absence of a control group.

\section{CONCLUSION}

The study shows that speech therapy combined with tDCS has better results in fluent aphasic patients whose initial treatment began within 30 days after stroke onset, and had AQ\% of $20 \%$ or more in the initial K-WAB evaluation. Initial severity of aphasia was strongly associated with improvement in speech function, with lower initial severity showing good responses. Hemorrhage 
rather than infarction was a better prognostic factor for speech function improvement, as it affected less on the cortical areas of brain related to language. Based on the results, combining tDCS with speech therapy or performing speech therapy alone to patients with lower initial severity was likely to show improved response to speech therapy. Combination of tDCS and speech therapy may be used in the treatment of aphasia but further large scale randomized trials are needed to confirm whether it is superior to conventional speech therapy.

\section{ACKNOWLEDGEMENTS}

This research was supported by research funds from Handok and Daewoong Pharmaceuticals Co., Ltd and SK Chemicals Ltd.

\section{REFERENCES}

1. Lim SJ, Kim HJ, Nam CM, Chang HS, Jang YH, Kim S, Kang HY. Socioeconomic costs of stroke in Korea: estimated from the Korea national health insurance claims database. J Prev Med Public Health 2009; 42: 251-260

2. Gresham GE, Fitzpatrick TE, Wolf PA, McNamara PM, Kannel WB, Dawber TR. Residual disability in survivors of stroke--the Framingham study. N Engl J Med 1975; 293: 954-956

3. Bersano A, Burgio F, Gattinoni M, Candelise L. Aphasia burden to hospitalised acute stroke patients: need for an early rehabilitation programme. Int J Stroke 2009; 4: 443-447

4. Tatemichi TK, Desmond DW, Stern Y, Paik M, Sano M, Bagiella E. Cognitive impairment after stroke: frequency, patterns, and relationship to functional abilities. J Neurol Neurosurg Psychiatry 1994; 57: 202207

5. Paolucci S, Antonucci G, Gialloreti LE, Traballesi M, Lubich S, Pratesi L, Palombi L. Predicting stroke inpatient rehabilitation outcome: the prominent role of neuropsychological disorders. Eur Neurol 1996; 36: 385-390

6. Laska AC, Hellblom A, Murray V, Kahan T, Von Arbin M. Aphasia in acute stroke and relation to outcome. J Intern Med 2001; 249: 413-422
7. Lazar RM, Antoniello D. Variability in recovery from aphasia. Curr Neurol Neurosci Rep 2008; 8: 497-502

8. Floel A, Rosser N, Michka O, Knecht S, Breitenstein C. Noninvasive brain stimulation improves language learning. J Cogn Neurosci 2008; 20: 1415-1422

9. Monti A, Cogiamanian F, Marceglia S, Ferrucci R, Mameli F, Mrakic-Sposta S, Vergari M, Zago S, Priori A. Improved naming after transcranial direct current stimulation in aphasia. J Neurol Neurosurg Psychiatry 2008; 79: 451-453

10. Gandiga PC, Hummel FC, Cohen LG. Transcranial DC stimulation (tDCS): a tool for double-blind shamcontrolled clinical studies in brain stimulation. Clin Neurophysiol 2006; 117: 845-850

11. Lazar RM, Minzer B, Antoniello D, Festa JR, Krakauer JW, Marshall RS. Improvement in aphasia scores after stroke is well predicted by initial severity. Stroke 2010; 41: 1485- 1488

12. Paolucci S, Matano A, Bragoni M, Coiro P, De Angelis D, Fusco FR, Morelli D, Pratesi L, Venturiero V, Bureca I. Rehabilitation of left brain-damaged ischemic stroke patients: the role of comprehension language deficits. A matched comparison. Cerebrovasc Dis 2005; 20: 400-406

13. Brust JC, Shafer SQ, Richter RW, Bruun B. Aphasia in acute stroke. Stroke 1976; 7: 167-174

14. Pedersen PM, Vinter K, Olsen TS. Aphasia after stroke: type, severity and prognosis. The Copenhagen aphasia study. Cerebrovasc Dis 2004; 17: 35-43

15. Lazar RM, Speizer AE, Festa JR, Krakauer JW, Marshall RS. Variability in language recovery after first-time stroke. J Neurol Neurosurg Psychiatry 2008; 79: 530534

16. Bakheit AM, Shaw S, Carrington S, Griffiths S. The rate and extent of improvement with therapy from the different types of aphasia in the first year after stroke. Clin Rehabil 2007; 21: 941-949

17. Hillis AE. The 'standard' for poststroke aphasia recovery. Stroke 2010; 41: 1316-1317

18. Nitsche MA, Nitsche MS, Klein CC, Tergau F, Rothwell JC, Paulus W. Level of action of cathodal DC polarisation induced inhibition of the human motor cortex. Clin Neurophysiol 2003; 114: 600-604

19. Keller SS, Crow T, Foundas A, Amunts K, Roberts N. Broca's area: nomenclature, anatomy, typology and asymmetry. Brain Lang 2009; 109: 29-48 
20. Bennett MR, Hacker PM. Language and cortical function: conceptual developments. Prog Neurobiol 2006; 80: $20-52$

21. Hong JH, Kim SH, Ahn SH, Jang SH. The anatomical location of the arcuate fasciculus in the human brain: a diffusion tensor tractography study. Brain Res Bull 2009; 80: 52-55

22. Kang EK, Sohn HM, Han MK, Kim W, Han TR, Paik NJ. Severity of post-stroke aphasia according to aphasia type and lesion location in Koreans. J Korean Med Sci 2010; 25: 123-127

23. Crinion JT, Leff AP. Recovery and treatment of aphasia after stroke: functional imaging studies. Curr Opin Neurol 2007; 20: 667-673

24. Breier JI, Juranek J, Maher LM, Schmadeke S, Men D, Papanicolaou AC. Behavioral and neurophysiologic response to therapy for chronic aphasia. Arch Phys Med Rehabil 2009; 90: 2026-2033

25. Harnish SM, Neils-Strunjas J, Lamy M, Eliassen JC. Use of fMRI in the study of chronic aphasia recovery after therapy: a case study. Top Stroke Rehabil 2008; 15: $468-483$
26. Szaflarski JP, Ball A, Grether S, Al-Fwaress F, Griffith NM, Neils-Strunjas J, Newmeyer A, Reichhardt R. Constraint-induced aphasia therapy stimulates language recovery in patients with chronic aphasia after ischemic stroke. Med Sci Monit 2008; 14: 243-250

27. Aftonomos LB, Appelbaum JS, Steele RD. Improving outcomes for persons with aphasia in advanced community-based treatment programs. Stroke 1999; 30: 1370-1379

28. Fertonani A, Rosini S, Cotelli M, Rossini PM, Miniussi C. Naming facilitation induced by transcranial direct current stimulation. Behav Brain Res 2010; 208: 311318

29. Baker JM, Rorden C, Fridriksson J. Using transcranial direct-current stimulation to treat stroke patients with aphasia. Stroke 2010; 41: 1229-1236

30. Fiori V, Coccia M, Marinelli CV, Vecchi V, Bonifazi S, Ceravolo MG, Provinciali L, Tomaiuolo F, Marangolo P. Transcranial direct current stimulation improves word retrieval in healthy and nonfluent aphasic subjects. J Cogn Neurosci 2011; 23: 2309-2323 